Pacific Journal of 


\title{
SUMS OF BOOLEAN SPACES REPRESENT EVERY GROUP
}

\author{
J. Adámek, V. Koubek and V. Trnková
}

For every Abelian group $(S,+)$ there exist Boolean - i.e., compact, 0-dimensional-topological spaces $X_{s}, s \in S$, such that $s+t=u$ if and only if $X_{u}$ is homeomorphic to the disjoint union of $X_{s}$ and $X_{t}$. The method of the proof of this theorem is topological, utilizing mostly properties of Čech-Stone compactifications of various spaces. A corollary, obtained from well-known dualities, is the representability of Abelian groups (in an analogous sense) by products of rings, lattices, Boolean algebras, Banach spaces or Banach algebras.

Let us recall the definition [11]:

Definition. Let $(S,+)$ be a commutative semigroup, let $K$ be a category with products $\times$ (or sums $\vee$ ) of finite collections of objects. A mapping

$$
r: S \longrightarrow \text { obj } K
$$

is called a representation of $S$ by products (or sums) in $K$ whenever

(i) if $s \neq s^{\prime}$, then $r(s)$ is not isomorphic to $r\left(s^{\prime}\right)$;

(ii) $r(s+t)$ is isomorphic to $r(s) \times r(t)$ (or $r(s) \vee r(t)$, respectively).

Many results concerning these representations can be, implicitly or explicitly, found in literature. Each finite cyclic group has a representation by products (or sums, which is the same in this case) in the category of Abelian groups [3]. Each semigroup on one generator has a representation by products in the category of modules over a suitable ring [5, 7]. Each semigroup on one generator and each Abelian group have representations by products in the category of topological spaces $[10,11]$ in the category of small categories [11], in a lot of subcategories of the category of graphs $[1,6]$, and unary universal algebras [2].

In the present note we generalize the following results of [4]: The group $C_{2}$ (i.e. the cyclic group of order 2) has a representation by products in the category of Boolean algebras or, which is the same, it has a representation by sums in the category of 0-dimensional compact Hausdorff spaces (i.e. Boolean spaces). In the present paper we prove that each Abelian group has such a representation. Thus, taking e.g. the additive group of all real numbers as the represented group, we obtain a Boolean space $X$ which "can be multiplied by 
any real number $\lambda^{\prime \prime}$ such that if $\lambda \neq \lambda^{\prime}$, then the spaces $\lambda X$ and $\lambda^{\prime} X$ are nonhomeomorphic, but $(\lambda+\mu) X$ is always homeomorphic to $\lambda X \vee \mu X$ (and $(\lambda \cdot \mu) X=\lambda(\mu X))$.

\section{The main theorem.}

Construction 1. Given a collection $\mathfrak{X}=\left\{X_{i} ; i \in I\right\}$ of compact Hausdorff spaces, denote by $\mathrm{V}_{i \in I} X_{i}$ their disjoint union, which is a space on the set $U\left(X_{i} \times\{i\}\right)$ with an obvious topology; denote by $s(\mathfrak{X})$ the Čech-Stone compactification $s(\mathfrak{X})=\beta\left(\vee X_{i}\right)$ (i.e., the sum in the category of compact Hausdorff spaces). Considering $I$ as a discrete space, we define a space $p(\mathfrak{X})$, which we obtain from $\beta I$ by replacing each $i \in I$ by the space $X_{i}: p(\mathfrak{X})$ is a space on the set $\cup\left(X_{i} \times\{i\}\right) \cup$ $(\beta I-I)$, whose topology on $X_{i} \times\{i\}$ is that of $X_{i}$ and whose quotient space under the mapping $f: p(\mathfrak{X}) \rightarrow \beta I$, which carries $X_{i} \times\{i\}$ to $\{i\}$ and leaves $\beta I-I$ unchanged, is just the space $\beta I$.

Notice that if $\mathfrak{X}$ is a collection of Boolean spaces then $p(\mathfrak{X})$ is also a Boolean space. Furthermore $\beta I-I$ is a subspace of $p(\mathfrak{X})$.

Let $\mathfrak{X}_{1}=\left\{X_{i} ; i \in I_{1}\right\}$ and $\mathfrak{X}_{2}=\left\{X_{i} ; i \in I_{2}\right\}$ be collections of compact spaces where $I_{1}, I_{2}$ are disjoint, and let $\mathfrak{X}=\left\{X_{i} ; i \in I_{1} \vee I_{2}\right\}$. Then $p\left(\mathfrak{X}_{1}\right) \vee p\left(\mathfrak{X}_{2}\right)=p(\mathfrak{X})$, which follows easily from the additivity of ČechStone compactifications.

LEMMA 2. Let $\mathfrak{X}=\left\{X_{2} ; \lambda \in L\right\}$ and $\mathfrak{Y}=\left\{Y_{\mu} ; \mu \in M\right\}$ be collections of compact Hausdorff spaces. Let $h$ be a homeomorphism of $p(\mathfrak{X})$ onto $p(\mathfrak{Y )}$ which maps $\beta L-L$ onto $\beta M-M$. Then there exist finite $L^{\prime} \subset L, M^{\prime} \subset M$ and a bijection $\varphi$ of $L-L^{\prime}$ onto $M-M^{\prime}$ such that, for each $\lambda \in L-L^{\prime}, h$ maps $X_{\lambda}$ onto $Y_{\varphi(\lambda)}$.

Proof. (a) For each $\lambda \in L$ (or $\mu \in M$ ) denote $k(\lambda)=\left\{\mu \in M ; h\left(X_{\lambda}\right) \cap\right.$ $\left.Y_{\mu} \neq \varnothing\right\}\left(\right.$ or $\left.m(\mu)=\left\{\lambda \in L ; h^{-1}\left(Y_{\mu}\right) \cap X_{\lambda}\right\} \neq \varnothing\right)$. Since $h$ maps $\beta L-L$ onto $\beta M-M$, each $k(\lambda)$ as well as each $m(\mu)$ is finite. Denote by $L_{1}$ (or $M_{1}$ ) the set of all $\lambda \in L$ (or $\mu \in M$ ) such that card $k(\lambda) \geqq 2$ (or card $m(\mu) \geqq 2$, respectively).

(b) We show that $L_{1}$ and $M_{1}$ are finite. Let us suppose that $L_{1}$ is infinite. Since, for each $\lambda \in L, \bigcup_{\mu \in k(\lambda)} m(\mu)$ is finite, we can find an infinite $L_{2} \subset L_{1}$ such that $k(\lambda) \cap k\left(\lambda^{\prime}\right)=\varnothing$ whenever $\lambda, \lambda^{\prime} \in L_{2}, \lambda \neq$ $\lambda^{\prime}$. For each $\lambda \in L_{2}$ choose different $\gamma_{\lambda}$, $\delta_{\lambda}$ in $k(\lambda)$. Denote by $C$ (or $D$ ) the closure of the set $\left\{\gamma_{\lambda} ; \lambda \in L_{2}\right\}$ (or $\left\{\delta_{\lambda} ; \lambda \in L_{2}\right\}$ ) in $\beta M$. Choose $x \in \bar{L}_{2}-L_{2}$, where $\bar{L}_{2}$ is the closure of $L_{2}$ in $\beta L$. Then necessarily $h(x) \in C$ as well as $h(x) \in D$, but $C \cap D=\varnothing$.

(c) Put $L^{\prime}=L_{1} \cup \bigcup_{\mu_{M_{1}}} m(\mu), M^{\prime}=M_{1} \cup \bigcup_{\lambda \in L_{1}} k(\lambda)$. Then $L^{\prime}, M^{\prime}$ are finite and if $\lambda \in L-L^{\prime}, k(\lambda)$ has precisely one element, say $\varphi(\lambda)$, and $m(\varphi(\lambda))=\{\lambda\}$. Since $h$ is a homeomorphism onto $p(\mathfrak{Y})$, it maps 
$X_{\lambda}$ onto $Y_{\varphi(\lambda)}$.

THEOREM 3. Each Abelian group has a representation by sums in the category of Boolean spaces and continuous mappings.

Proof. (a) Let $n$ be a natural number, $C_{n}$ be a cyclic group of order $n$, its elements are considered as $\overline{0}, \overline{1}, \cdots, \overline{n-1}$, where $\bar{k}$ is the set of all integers congruent to $k$ modulo $n$. Let $m$ be an infinite cardinal number. We construct a special representation $r_{m}$ of $C_{n}$. Denote by $P_{m}$ the one-point compactification of a discrete space of the cardinality $m$. If $k \in\{0, \cdots, n-1\}$, denote by $\mathfrak{X}_{k}$ the collection $\left\{X_{\lambda} ; \lambda \in N\right\}$, where $N=\{1,2, \cdots\}$,

$$
\begin{aligned}
X_{1} & =P_{m} \vee P_{m} \vee \cdots \vee P_{m} k \text {-times , }\left(X_{1}=\varnothing \text { if } k=0\right), \\
\lambda \neq 1 & X_{\lambda}=P_{m} \vee P_{m} \vee \cdots \vee P_{m} n \text {-times . }
\end{aligned}
$$

Put $r_{m}(\bar{k})=p\left(\mathfrak{X}_{k}\right)$. We show that $r_{m}$ is really a representation. Clearly, $r_{m}\left(\bar{k}_{1}\right) \vee r_{m}\left(\bar{k}_{2}\right)$ is homeomorphic to $r_{m}\left(\overline{k_{1}+k_{2}}\right)$. Let us suppose that for some $0 \leqq k_{1}<k_{2} \leqq n-1$ there exists a homeomorphism $h$ of $r_{m}\left(\bar{k}_{1}\right)$ onto $r_{m}\left(\bar{k}_{2}\right)$. We show that $h(\beta N-N)=(\beta N-N)$. In fact, the other points of $r_{m}\left(\bar{k}_{1}\right)$ and of $r_{m}\left(\bar{k}_{2}\right)$ are either isolated, or they have a neighbourhood $\mathscr{U}$ such that card $\mathscr{U}=m$ and for any other neighbourhood $\mathscr{\mathscr { C }} \mathscr{\mathscr { C }}-\mathscr{V}$ is finite. None of the points of $\beta N-N$ has this property. Now, use Lemma 2.

(b) Let $I$ be a subset of the set of all natural numbers. Let $\left\{C_{n} ; n \in I\right\}$ be a collection of finite cyclic groups $\left(C_{n}\right.$ has the order $\left.n\right)$. Let $C$ be their product. Let $M=\left\{m_{i} ; i \in I\right\}$ be a collection of infinite cardinal numbers such that $m_{i} \neq m_{i}$, whenever $i \neq i^{\prime}$. We construct a special representation $r_{M}$ of $C$. If $x=\left\{x_{i} ; i \in I\right\}$ is a point of $C$ denote $\mathfrak{X}_{x}=\left\{r_{m_{i}}\left(x_{i}\right) ; i \in I\right\}$. Put $r_{M}(x)=s\left(\mathfrak{X}_{x}\right)$. We show that $r_{M}$ is really a representation. $r_{M}(x) \vee r_{M}\left(x^{\prime}\right)$ is clearly homeomorphic to $r_{M}\left(x+x^{\prime}\right)$. If $x, x^{\prime} \in C$ and $x \neq x^{\prime}$, there exists $i \in I$ such that $r_{m_{i}}\left(x_{i}\right)$ is not homeomorphic to $r_{m_{i}}\left(x_{i}^{\prime}\right)$. Since $m_{i} \neq m_{i^{\prime}}$ whenever $i \neq i^{\prime}, r_{M}(x)$ cannot be homeomorphic to $r_{M}\left(x^{\prime}\right)$.

(c) Let $p$ be a positive prime. We denote by $R_{p}$ the additive group of all $p$-adic rational numbers, i.e. numbers of the form $k / p^{n}$, $k$ an integer, $n$ a natural number. Denote by $L$ the set of all primes bigger than $p . R_{p}$ is isomorphic to a subgroup of $\times_{k \in L} C_{k}$. There exists a one-to-one homomorphism $\varphi: R_{p} \rightarrow \mathrm{X}_{k \in L} C_{k}$ such that $\varphi(1)=$ $\{1,1, \cdots\}$.

(d) Let $p$ be a positive prime. As usual, we denote by $C_{p^{\infty}}$ the group $R_{p}$ modulo integers, elements of $C_{p^{\infty}}$ are considered as $\bar{x}$, where $x$ is a $p$-adic rational number, $0 \leqq x<1$, and $\bar{x}$ is the set of all $p$ adic rational numbers congruent to $x$ modulo integers. We construct 
a special representation of $C_{p^{\infty}}$. Let $L, \varphi, M, r_{M}$ have the same meaning as in (b), (c). If $\bar{x} \in C_{p^{\infty}}, 0 \leqq x<1$, put $\mathfrak{X}_{x}=\left\{X_{n} ; n \in N\right\}, X_{1}=$ $r_{M}(\varphi(x)), X_{n}=r_{M}(\varphi(1))$ for all $n>1$. Put $q_{M}(\bar{x})=p\left(\mathfrak{X}_{x}\right)$. We show that $q_{M}$ is a representation of $C_{p^{\infty}} . q_{M}(\bar{x}) \vee q_{M}\left(\bar{x}^{\prime}\right)$ is homeomorphic to $q_{M} \overline{\left(x+x^{\prime}\right)}$, or to $g_{M}\left(\overline{\left.x+x^{\prime}-1\right)}\right.$ whenever $x+x^{\prime} \geqq 1$ because $r_{M}(\varphi(x)) \vee r_{M}\left(\varphi\left(x^{\prime}\right)\right)$ is homeomorphic to $r_{M}\left(\varphi\left(x+x^{\prime}\right)\right)$. Let $\bar{x}, \bar{x}^{\prime}$ be $p$-adic rational numbers, $0 \leqq x<x^{\prime}<1$. Let us suppose that there exists a homeomorphism $h$ of $q_{M}(\bar{x})$ onto $q_{M}\left(\bar{x}^{\prime}\right)$. Let us prove that $h(\beta N-N)=\beta N-N$. As follows from the construction of $q_{M}, \beta N-N$ can be characterized by neighbourhoods of points: let $z$ be a point of $q_{M}(\bar{x})$ (proceed analogously for $q_{M}\left(\bar{x}^{\prime}\right)$ ). Then $z \in \beta N-N$ if and only if every neighbourhood $\mathscr{U}$ of $z$ contains a subspace of the form $\bigvee_{k \in L} P\left(m_{k}\right)$, where each $P\left(m_{k}\right)$

(1) is homeomorphic to the one-point compactification of a discrete space of power $m_{k}$,

(2) is not contained in any subspace of $q_{M}(\bar{x})$, homeomorphic to the one-point compactification of a discrete space of power bigger than $m_{k}$.

Proof. Let $z \in \beta N-N$, let $\mathscr{U}$ be its neighbourhood. Then clearly $\mathscr{U}$ has an open-and-closed subspace, homeomorphic to $r_{M}(\varphi(1))$ (recall that $X_{n}=r_{M}(\varphi(1))$ with one exception). Thus, it suffices to notice that $r_{M}(\varphi(1))$ has a subspace with the mentioned properties. On the other hand, if $z \notin \beta N-N$, then $z \in X_{n} \times\{n\}$ for some $n$ and it is easy to see, that there exists a neighbourhood of $z$ which has no subspace of the considered form.

Therefore clearly $h(\beta N-N)=\beta N-N$. Now apply Lemma 2: there exist finite sets $A, B \subset N$ with $1 \in A \cap B$ and a bijection $\psi: N-A$ $\rightarrow N-B$ with $h\left(X_{n}\right)=X_{\psi(n)}$ for all $n \in N-A$. Then $h\left(\mathrm{U}_{n \in A}\left(X_{n} \times\right.\right.$ $\{n\}))=\bigcup_{m \in B}\left(X_{m} \times\{m\}\right)$, which is a contradiction, since then $r_{M}(\varphi(x+$ $a-1)$ ) is homeomorphic to $r_{M}\left(\varphi\left(x^{\prime}+b-1\right)\right)$, where $a=\operatorname{card} A, b=$ card $B$, and so $x-x^{\prime}$ is an integer.

(e) Let $\left\{p_{i} ; i \in I\right\}$ be a collection of positive primes. For each $i \in I$ denote by $L_{i}$ the set of all primes bigger than $p_{i}$. Let $\left\{m_{i, k} ; i \in I\right.$, $\left.k \in L_{i}\right\}$ be a collection of pairwise distinct infinite cardinals. Denote $M_{i}=\left\{m_{i, k} ; k \in L_{i}\right\}$. Let $q_{M_{i}}$ be the representation of $C_{p_{i}}$ constructed in (d). We construct a representation $r$ of $C=X_{i \in I} C_{p_{i}^{\infty}}$. If $\bar{x}=\left\{\bar{x}_{i}\right.$; $i \in I\} \in C$, i.e. $\bar{x}_{i} \in C_{p_{i}}^{\infty}$ for each $i$, put $r(\bar{x})=s\left\{\left\{q_{m_{i}}\left(x_{i}\right) ; i \in I\right\}\right)$. The proof that $r$ is really a representation is analogous to $(b)$.

(f) For any Abelian group $G$ there exists a collection $\left\{p_{i} ; i \in I\right\}$ of primes such that $G$ is isomorphic to a subgroup of $X_{i \in I} C_{p_{i}^{\infty}}$. This follows from Theorem 5.1 in [9].

II. Applications. First, since each compact Hausdorff space 
induces a unique uniform and proximity structure, the above result yields: every Abelian group has a representation by sums of

(1) uniform spaces;

(2) proximity spaces.

Second, since the category of Boolean spaces is dual to the category of Boolean algebras, each Abelian group has a representation by products in it.

Now, let $X$ be a compact Hausdorff space. Denote by $C(X)$ the set of all real continuous functions on $X . \quad C(X)$ may be structured in a lot of ways, for example as a ring or a lattice etc. Thus, $C$ may be considered as a contravariant functor of the category of compact Hausdorff spaces into the category of rings or lattices and so on. We can apply the result about representation of any Abelian groups by sums of Boolean spaces and obtain results about representation by products if $C(X)$ is structured such that

(a) $C$ turns sums into products;

(b) if $X$ and $X^{\prime}$ are nonhomeomorphic, then $C(X)$ and $C\left(X^{\prime}\right)$ are nonisomorphic.

The condition (a) is usually satisfied evidently; the condition (b) are often classical theorems.

We show some examples:

(1) commutative rings (with unit), (a) is satisfied, evidently; (b) is the classical Gelfand-Kolmogoroff theorem;

(2) commutative monoids. Here we consider the multiplicative semigroup of the real continuous functions, (a) is evident, (b) see [8];

(3) lattices, (a) is evident, (b) follows from the Kaplansky theorem;

(4) linear lattices (and linear homomorphism), (a) is evident, (b) follows also from the Kaplansky theorem;

(5) Banach spaces. Here, we consider linear contractions as morphisms (i.e. bounded linear operators with norm $\leqq 1$ ). Then (a) is evident, (b) follows from the classical Banach-Stone theorem;

(6) Banach algebras (morphisms are bounded homomorphisms with norm $\leqq 1$ ). (a) is evident, (b) follows also from the BanachStone theorem.

On the other hand (b) is not satisfied if we consider $C(X)$ only as a linear space. It is very easy to verify that no nontrivial group has a representation by products in the category of linear spaces.

\section{REFERENCES}

1. J. Adámek and V. Koubek, Products of graphs as a representation of semigroups, Recent advances in graph theory, Proc. of Symp., Prague (1974), 13-16.

2. - A representation of semigroups by products of unary algebras and relations, to appear. 
3. A. L. Corner, On a conjecture of Pierce concerning direct decomposition on Abelian groups, Proc. of the Coll. on Abelian groups, Tihany (1963), 43-48.

4. P. R. Halmos, Lectures on Boolean algebras, Princeton, Van Nostrand Math. Studies, 1963.

5. P. M. Kohn, Some remarks on the invariance bases property, Topology, 5 (1966), 215-228.

6. V. Koubek, J. Nešetřil and V. Rödl, Representing groups and semigroups by products in categories of relations, Alg. Universalis, 4 (1974), 336-341.

7. W. G. Leavitt, The modul type of ring, Trans. Amer. Math. Soc., 103 (1962), 113-130.

8. A. N. Milgram, Multiplicative semigroups of continuous functions, Duke Math. J., 16 (1949), 337-383.

9. B. M. Schein, Homomorphisms and subdirect decompositions of semigroups, Pacific J. Math., 17 (1966), 529-547.

10. V. Trnková, $X^{m}$ is homeomorphic to $X^{n}$ iff $m \sim n$ where $\sim$ is a congruence on natural numbers, Fund. Math., 80 (1973), 51-56.

11. V. Trnková, $A$ representation of semigroups by products in a category, J. of Algebra, 34 (1975), 191-204.

Received September 6, 1974 and in revised form February 14, 1975.

Technical University, Prague

AND

Charles University, Prague 


\section{PACIFIC JOURNAL OF MATHEMATICS}

\section{EDITORS}

RICHARD ARENS (Managing Editor) University of California

Los Angeles, California 90024

\section{R. A. BEAUMONT}

University of Washington Seattle, Washington 98105
J. DugundjI Department of Mathematics University of Southern Californı Los Angeles, California 90007

D. Gilbarg AND J. Milgram Stanford University Stanford, California 94305

\section{ASSOCIATE EDITORS}

E. F. BECKENBACH

B. H. NeUMANN

F. WOLF

K. YosHidA

\section{SUPPORTING INSTITUTIONS}

UNIVERSITY OF BRITISH COLUMBIA CALIFORNIA INSTITUTE OF TECHNOLOGY UNIVERSITY OF CALIFORNIA MONTANA STATE UNIVERSITY UNIVERSITY OF NEVADA NEW MEXICO STATE UNIVERSITY OREGON STATE UNIVERSITY UNIVERSITY OF OREGON OSAKA UNIVERSITY

\author{
UNIVERSITY OF SOUTHERN CALIFORNIA \\ STANFORD UNIVERSITY \\ UNIVERSITY OF TOKYO \\ UNIVERSITY OF UTAH \\ WASHINGTON STATE UNIVERSITY \\ UNIVERSITY OF WASHINGTON \\ AMERICAN MATHEMATICAL SOCIETY \\ NAVAL WEAPONS CENTER
}




\section{Pacific Journal of Mathematics}

\section{Vol. 61, No. $1 \quad$ November, 1975}

Jiří Adámek, V. Koubek and Věra Trnková, Sums of Boolean spaces represent every

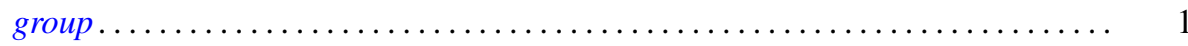

Richard Neal Ball, Full convex l-subgroups and the existence of $a^{*}$-closures of

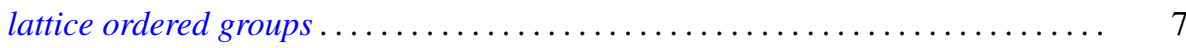

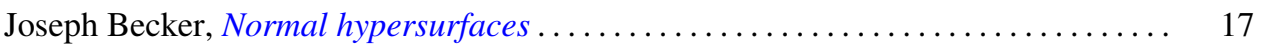

Gerald A. Beer, Starshaped sets and the Hausdorff metric . . . . . . . . . . . . . 21

Dennis Dale Berkey and Alan Cecil Lazer, Linear differential systems with

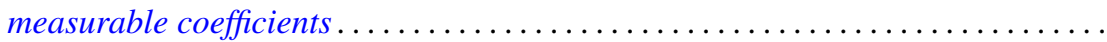

Harald Boehme, Glättungen von Abbildungen 3-dimensionaler

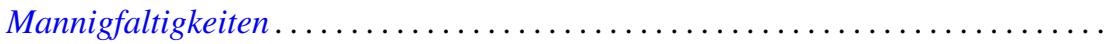

Stephen LaVern Campbell, Linear operators for which $T^{*} T$ and $T+T^{*}$

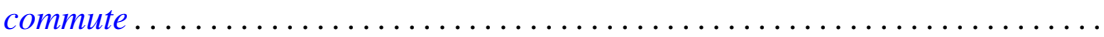

H. P. Dikshit and Arun Kumar, Absolute summability of Fourier series with

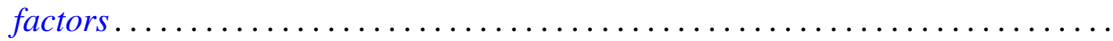

Andrew George Earnest and John Sollion Hsia, Spinor norms of local integral

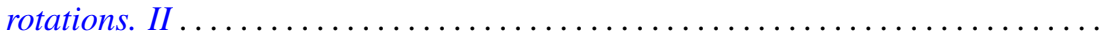

Erik Maurice Ellentuck, Semigroups, Horn sentences and isolic structures .........

Ingrid Fotino, Generalized convolution ring of arithmetic functions . . . . . . . . . . .

Michael Randy Gabel, Lower bounds on the stable range of polynomial rings .......

Fergus John Gaines, Kato-Taussky-Wielandt commutator relations and

characteristic curves

Theodore William Gamelin, The polynomial hulls of certain subsets of $C^{2}$

R. J. Gazik and Darrell Conley Kent, Coarse uniform convergence spaces. . .

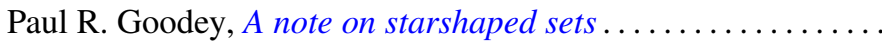

Eloise A. Hamann, On power-invariance

M. Jayachandran and M. Rajagopalan, Scattered compactification for $N \cup\{P\}$. . .

V. Karunakaran, Certain classes of regular univalent functions .

John Cronan Kieffer, A ratio limit theorem for a strongly subadditive set function in a locally compact amenable group .................

Siu Kwong Lo and Harald G. Niederreiter, Banach-Buck measure, density, and uniform distribution in rings of algebraic integers ........

Harold W. Martin, Contractibility of topological spaces onto metric spaces ....

Harold W. Martin, Local connectedness in developable spaces .

A. Meir and John W. Moon, Relations between packing and covering numbers of a tree.

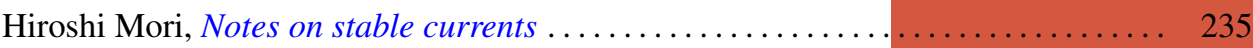

Donald J. Newman and I. J. Schoenberg, Splines and the logarithmic function . . . . 241

M. Ann Piech, Locality of the number of particles operator....

Fred Richman, The constructive theory of $K T$-modules .......

Gerard Sierksma, Carathéodory and Helly-numbers of

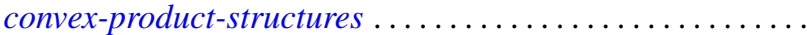

Raymond Earl Smithson, Subcontinuity for multifunctions .... . . 